\title{
New Adaptive Mode of Operation for MEMS Gyroscopes
}

\author{
Sungsu Park ${ }^{1}$ and Roberto Horowitz ${ }^{2}$ \\ ${ }^{1}$ Department of Aerospace Engineering \\ Sejong University, Seoul, Korea \\ ${ }^{2}$ Department of Mechanical Engineering \\ University of California, Berkeley \\ Berkeley, CA 94720 \\ (Email)sungsu@sejong.ac.kr; horowitz@me.berkeley.edu
}

\begin{abstract}
This paper presents a new adaptive operation strategy that can identify and, in an adaptive fashion, compensate for most fabrication defects and perturbations affecting the behavior of a MEMS zaxis gyroscope. The convergence and resolution analysis presented in paper shows that the proposed adaptive controlled scheme offers several advantages over conventional modes of operation. These advantages include a larger operational bandwidth, absence of zero-rate output, self-calibration and a large robustness to parameter variations, which are caused by fabrication defects and ambient conditions.
\end{abstract}




\section{INTRODUCTION}

Gyroscopes are commonly used sensors for measuring angular velocity in many areas of applications such as navigation, homing, and control stabilization. Although, conventional rotating wheel, fiber optic and ring laser gyroscopes have dominated a wide range of applications, they are too large and, most often too expensive to be used in most emerging applications.

Recent advances in micro-machining technology have made the design and fabrication of MEMS (Micro-Electro-Mechanical Systems) gyroscopes possible. These devices are several orders of magnitude smaller than conventional mechanical gyroscopes, and can be fabricated in large quantities by batch processes. Thus, there is great potential to significantly reduce their fabrication cost. The emergence of MEMS gyroscopes is opening up new market opportunities and applications in the area of low-cost to medium performance inertial devices, including consumer electronics such as virtual reality, video games, 3D mouse and camcorder image stabilization; automotive applications such as ride stabilization, rollover detection and other vehicle safety systems; GPS augmentation such as MEMS inertial navigation sensor imbedded GPS; as well as a wide range of new military applications such as micro airplanes and satellite controls.

The design and fabrication of MEMS gyroscopes has been the subject of extensive research over the past few years. [1] contains a comprehensive review of previous efforts in developing high quality cost-effective gyroscopes. Also noted in [1] is the fact that the cost of MEMS gyroscopes is decreasing while their accuracy is continuously being improved. Existing forecasts have indicated that this trend will continue. 
All MEMS gyroscopes are laminar vibratory mechanical structures fabricated on polysilicon or crystal silicon. Common fabrication steps include bulk micromachining, wafer-to-wafer bonding, surface micromachining, and high aspect ratio micromachining. Each of these fabrication steps involves multiple process steps such as deposition, etching and patterning of materials. In practice, small imperfections always occur during the fabrication process. Depending on the technology used, different numbers of steps may be involved in the fabrication of a MEMS gyroscope, and different fabrication tolerances can be achieved. Generally, every fabrication step contributes to imperfections in the gyroscope [2]. Fabrication imperfections that produce asymmetric structures, misalignment of actuation mechanism and deviations of the center of mass from the geometric center, result in undesirable, systematic perturbations in the form of mechanical and electrostatic forces, which degrade the performance of a gyroscope. Resolution, drift, scale factor and zero-rate output (ZRO) are important factors that determine the gyroscope performance [1,3]. Geometrical imperfections as well as electrical coupling cause degradation of these performance indexes. As a consequence, some kind of control is essential for improving the performance and stability of MEMS gyroscopes, by effectively canceling "parasitic" effects. Traditionally, mechanical or electrical balancing has been used to cancel parasitic effects [4-6]. Although this procedure reduces the effect of a certain amount of imperfections, it is time consuming, expensive and difficult to perform on small, nail-size ( $\mathrm{mm}$ level) gyroscopes. Moreover, this procedure is performed for a single operating condition. Variations in temperature and pressure may take place during the operation of the gyroscope, which affect parasitic effects. 
The control law for MEMS gyroscopes may be designed so as to estimate the angular rate directly or indirectly, depending on the operation mode. The operation mode is the operating topology of the gyroscope regarding its electro-mechanical design, its internal dynamics, how to manage its imperfections and environment variations and what sensing resources are to use to measure the gyroscope motion. The performance and accuracy of the gyroscope depends on the operation mode and corresponding control law design. Controls for MEMS gyroscopes are still theoretically immature. In terms of automatic controls, two different types of controllers have been proposed for conventional mode of operation in the literature. One is a Kalman filter based preview control [7] and the other is a recently published force-balancing feedback control scheme using sigma-delta modulation [8]. Although these feedback control techniques increase the bandwidth and dynamic range of the gyroscope beyond the open-loop mode of operation, they still are sensitive to parameter variations such as damping, spring constant and quadrature error variations, produce ZRO and require tedious calibrations.

The objective of this paper is to develop a new gyroscope operation mode, and to formulate a corresponding control algorithm that is well suited for the on-line compensation of imperfects and to operate in varying environments that affect the behavior of a MEMS gyroscope. The adaptive controlled gyroscope is self-calibrating, compensates for friction forces, and fabrication imperfections which normally cause quadrature errors, and produces an unbiased angular velocity measurement that has no ZRO.

In the next section, the dynamics of MEMS gyroscopes is developed and analyzed, by accounting for the effect of fabrication imperfections. The conventional operation modes 
such as open-loop and closed-loop modes, is reviewed in section III. An adaptive control approach for measuring angular rate is proposed as a new operation mode, and the convergence and resolution analysis of the proposed adaptive controlled gyroscope is presented in sections IV and V. Finally, computer simulations are performed in section VI.

\section{DYNAMICS OFMEMS GYROSCOPES}

Common MEMS vibratory gyroscope configurations include a proof mass suspended by spring suspensions, and electrostatic actuations and sensing mechanisms for forcing an oscillatory motion and sensing the position and velocity of the proof mass. These mechanical components can be modeled as a mass, spring and damper system. The mass in a vibratory gyroscope is generally constrained to move either linearly or angularly. In this paper, only linear vibratory gyroscopes are discussed. However, most of the results of this paper are applicable to angular vibratory gyroscopes as well.

Figure 1 shows a simplified model of a MEMS gyroscope having two degrees of freedom in the associated Cartesian reference frames. Assuming that the motion of the proof mass is constrained to be only along the $x-y$ plane by making the spring stiffness in the $z$ direction much larger than in the $x$ and $y$ directions, the measured angular rate is almost constant over a long enough time interval, and linear accelerations are cancelled out, either as an offset from the output response or by applying counter-control forces, the equation of motion of a gyroscope is simplified as follows. 


$$
\begin{aligned}
& m \ddot{x}+d_{1} \dot{x}+\left(k_{1}-m\left(\Omega_{y}^{2}+\Omega_{z}^{2}\right)\right) x+m \Omega_{x} \Omega_{y} y=\tau_{x}+2 m \Omega_{z} \dot{y} \\
& m \ddot{y}+d_{2} \dot{y}+\left(k_{2}-m\left(\Omega_{x}^{2}+\Omega_{z}^{2}\right)\right) y+m \Omega_{x} \Omega_{y} x=\tau_{y}-2 m \Omega_{z} \dot{x}
\end{aligned}
$$

where $x$ and $y$ are the coordinates of the proof mass relative to the gyro frame, $d_{1,2}, k_{1,2}$ are damping and spring coefficients, $\Omega_{x, y, z}$ are the angular velocity components along each axis of the gyro frame, and $\tau_{x, y}$ are control forces. The two last terms in Eq. (1), $2 m \Omega_{z} \dot{x}$ and $2 m \Omega_{z} \dot{y}$, are due to the Coriolis forces and are the terms which are used to measure the angular rate $\Omega_{z}$.

As seen in (1), in an ideal gyroscope, only the component of the angular rate along the $z$ axis, $\Omega_{z}$, causes a dynamic coupling between the $x$ and $y$ axes, under the assumption that $\Omega_{x}^{2} \approx \Omega_{y}^{2} \approx \Omega_{x} \Omega_{y} \approx 0$. In practice, however, small fabrication imperfections always occur, and also cause dynamic coupling between the $x$ and $y$ axes through the asymmetric spring and damping terms. These are major factors which limit the performance of MEMS gyroscopes. Taking into account fabrication imperfections, the dynamic equations (1) are modified as follows [9].

$$
\begin{aligned}
& m \ddot{x}+d_{x x} \dot{x}+d_{x y} \dot{y}+k_{x x} x+k_{x y} y=\tau_{x}+2 m \Omega_{z} \dot{y} \\
& m \ddot{y}+d_{x y} \dot{x}+d_{y y} \dot{y}+k_{x y} x+k_{y y} y=\tau_{y}-2 m \Omega_{z} \dot{x}
\end{aligned}
$$

Equation (2) is the governing equation for a $z$-axis MEMS gyroscope. Fabrication imperfections contribute mainly to the asymmetric spring and damping terms, $k_{x y}$ and $d_{x y}$. Therefore these terms are unknown, but can be assumed to be small. The $x$ and $y$ axes spring and damping terms are mostly known, but have small unknown variations from their nominal values. The proof mass can be determined very accurately. The components of 
angular rate along $x$ and $y$ axes are absorbed as part of the spring terms as unknown variations. Note that the spring coefficients $k_{x x}$ and $k_{y y}$ also include the electrostatic spring softness.

Non-dimensionalizing the equations of motion of a gyroscope is useful because the numerical simulation is easy, even under the existence of large two time-scales differences in gyroscope dynamics. One time scale is defined by the resonant natural frequency of the gyroscope, $\sqrt{k_{x x} / m}$, the other by the applied angular rate $\Omega_{z}$. Nondimensionalization also produces a unified mathematical formulation for a large variety of gyroscope designs. In this paper, controllers will be designed based on non-dimensional equations. The realization to a dimensional control for the specific gyroscope can be easily accomplished by multiplying the dimensionalizing parameters by the non-dimensional controller parameters. Based on $m, q_{0}$ and $\omega_{0}$, which are a reference mass, length and natural resonance frequency respectively, where $m$ is a proof mass of the gyroscope, the nondimensionalization of (2) can be done as follows:

$$
\begin{aligned}
& \ddot{x}+\frac{\omega_{x}}{Q_{x}} \dot{x}+d_{x y} \dot{y}+\omega_{x}^{2} x+\omega_{x y} y=\tau_{x}+2 \Omega_{z} \dot{y} \\
& \ddot{y}+d_{x y} \dot{x}+\frac{\omega_{y}}{Q_{y}} \dot{y}+\omega_{x y} x+\omega_{y}^{2} y=\tau_{y}-2 \Omega_{z} \dot{x}
\end{aligned}
$$

where $Q_{x}$ and $Q_{y}$ are respectively the $x$ and $y$ axis quality factor, $\omega_{x}=\sqrt{k_{x x} /\left(m \omega_{0}^{2}\right)}$, $\omega_{y}=\sqrt{k_{y y} /\left(m \omega_{0}^{2}\right)} \quad, \quad \omega_{x y}=k_{x y} /\left(m \omega_{0}^{2}\right) \quad, \quad d_{x y} \leftarrow d_{x y} /\left(m \omega_{0}\right) \quad, \quad \Omega_{z} \leftarrow \Omega_{z} / \omega_{0}$, $\tau_{x} \leftarrow \tau_{x} /\left(m \omega_{0}^{2} q_{0}\right)$ and $\tau_{y} \leftarrow \tau_{y} /\left(m \omega_{0}^{2} q_{0}\right)$ 
The natural frequency of the $x$ or $y$ axis can be used to define the nondimensionalizing parameter $\omega_{0}$. Since the usual displacement range of the MEMS gyroscope in each axis is sub-micrometer level, it is reasonable to choose $1 \mu \mathrm{m}$ as a reference length $q_{0}$. Considering that the usual natural frequency of each of the axis of a vibratory MEMS gyroscope is in the $\mathrm{KHz}$ range, while the applied angular rate may be in the degrees per second or degrees per hour range, the non-dimensional angular rate that we want to estimate is respectively in the range of $10^{-4}$ or $10^{-10}$.

\section{CONVENTIONAL MOde OF OPERATION}

The conventional mode of operation reduces to driving one of the modes of the gyroscope into a known oscillatory motion and then detecting the Coriolis acceleration coupling along the sense mode of vibration, which is orthogonal to the driven mode. The response of the sense mode of vibration provides information about the applied angular velocity. More specifically, the proof mass is driven into a constant amplitude oscillatory motion along the $x$-axis (drive axis) by the $x$-axis control $\tau_{x}$. When the gyroscope is subjected to an angular rotation, a Coriolis inertial specific force, $-2 \Omega_{z} \dot{x}$, is generated along the $y$-axis (sense axis), whose magnitude is proportional to the oscillation velocity of the drive axis and the magnitude of $z$-axis component of angular rate. This force excites the proof mass into an oscillatory motion along the $y$-axis, and its magnitude is amplified according to the mechanical quality factor ( $Q$-factor). Mathematically speaking, the governing equation for the conventional mode of operation is described as follows: 


$$
\begin{aligned}
& x=X_{0} \sin \left(\omega_{x} t\right) \\
& \ddot{y}+\frac{\omega_{y}}{Q_{y}} \dot{y}+\omega_{y}^{2} y=\tau_{y}-\omega_{x y} x-\left(d_{x y}+2 \Omega_{z}\right) \dot{x}
\end{aligned}
$$

The conventional mode of operation is classified into the open-loop mode and the closedloop mode. The main difference between the closed-loop and open-loop mode of operation lies in that in the former the displacement of the sense axis is controlled to zero, while in the latter it is measured.

Most MEMS gyroscopes are currently operated in the open-loop mode. The main advantage of open loop mode of operation is that circuitry used for the operation of gyroscope in this mode is simpler than in the other modes, since there is no control action in the sense axis. Thus, this mode can be implemented relatively easily and cheaply. However, under an open-loop mode of operation, the gyroscope's angular rate scale factor is very sensitive, and not constant over any appreciable bandwidth, to fabrication defects and environment variations. Therefore, the application areas for the open-loop mode are limited to those which require low-cost and low-performance gyroscopes.

In contrast to the open-loop mode of operation, in the closed-loop mode of operation, the sense amplitude of oscillation is continuously monitored and driven to zero. As a consequence, the bandwidth and dynamic range of the gyroscope can be greatly increased beyond what can be achieved with the open-loop mode of operation. However, under conventional closed-loop mode of operatio n, it is difficult to ensure a constant noise performance, in the face of environment variations such as temperature changes, unless an on-line mode tuning scheme is included. Moreover, there are practical difficulties in 
designing a feedback controller so that the closed-loop system is stable and sufficiently robust, for gyroscopes with high $Q$ systems. Therefore, the application areas for conventional closed-loop mode of operation are those which requires medium-cost and medium-performance (large bandwidth but limited resolution) gyroscopes.

Both the open-loop and closed-loop modes are inherently sensitive to some types of fabrication imperfections which can be modeled as the cross-damping term $d_{x y}$, which produce ZRO.

The detrimental effect of the asymmetric damping term $d_{x y}$ on gyroscope performance has not been considered by many researchers so far. However, its effect should not be underestimated. For example, using typical conventional gyroscope parameters adopted from Clark [4], various angular rate equivalent tilt angles $\kappa$ between the principal and physical damping axes yield Table 1 . The values of $\omega_{x}=10^{5} \mathrm{rad} / \mathrm{sec}, Q_{x}=10^{4}$ and $Q_{y}=10^{3}$ were used in calculating this table. Moreover, with the conventional modes of operation, it is also very difficult to identify and compensate for all fabrication imperfections in an on-line fashion, due to the simple internal dynamics of the gyroscope when is operating under these modes. One solution to achieve on-line compensation of fabrication imperfections may be to create a richer gyroscope dynamics than can be achieved in the conventional modes of operation. This idea led us to formulate a new operation strategy in which the two oscillatory modes of the z-axis gyroscope are not matched. 


\begin{tabular}{|c|c|}
\hline$\kappa(\operatorname{deg})$ & $\Omega_{z}$ \\
\hline 1.00 & $45.0 \mathrm{deg} / \mathrm{sec}$ \\
\hline 0.022 & $1.0 \mathrm{deg} / \mathrm{sec}$ \\
\hline $1 \times 10^{-5}$ & $1.6 \mathrm{deg} / \mathrm{hour}$ \\
\hline
\end{tabular}

Table 1. Angular rate equivalent tilt angle $\kappa$ between the principal and physical damping axes

\section{NEW AdAPTIVE MODE OF OPERATION}

This section proposes a new operating strategy for MEMS gyroscopes, which will be referred to as the adaptive mode of operation. Its aim is to achieve (1) on-line compensation of fabrication imperfections, (2) closed-loop identification of the angular rate, (3) to attain a large bandwidth and dynamic range, and (4) self-calibration operation. Proposed adaptive mode of operation will operate based on observer-based adaptive control algorithm which needs only position measurements of the proof mass of the gyroscope. Since observer-based adaptive control is the extension of the adaptive control based on velocity measurement, we first briefly present basic idea and control algorithm of it. 


\section{A. Velocity Measurement-Based Adaptive Control}

The basic idea of the adaptive control approach is to treat the angular rate, along with the effect of fabrication defects, as an unknown gyroscope parameter, which must be estimated using a parameter adaptation algorithm (PAA).

The adaptive control problems of the gyroscope is formalized as follows: given the equation with unknown constant parameters $D, K$ and $\Omega$,

$$
\ddot{q}+D \dot{q}+K q=\tau-2 \Omega \dot{q}
$$

where

$$
\begin{aligned}
& q=\left[\begin{array}{l}
x \\
y
\end{array}\right] \quad \tau=\left[\begin{array}{l}
\tau_{x} \\
\tau_{y}
\end{array}\right] \quad \Omega=\left[\begin{array}{cc}
0 & -\Omega_{z} \\
\Omega_{z} & 0
\end{array}\right] \\
& D=\left[\begin{array}{ll}
d_{x x} & d_{x y} \\
d_{x y} & d_{y y}
\end{array}\right] \quad K=\left[\begin{array}{ll}
\omega_{x}^{2} & \omega_{x y} \\
\omega_{x y} & \omega_{y}^{2}
\end{array}\right]
\end{aligned}
$$

determine the control law $\tau$ based on measuring $q$ and $\dot{q}$, such that the dynamic range is constrained within an intended region and $\Omega$ is estimated correctly. With this kind of problem formulation, we treat the gyroscope as a multi-dimensional dynamic device.

Like in other adaptive control problems, the persistent excitation condition is an important factor to estimate the angular rate correctly. To solve this problem, a trajectory following approach is used. The reference trajectory that the gyroscope must follow is generated such that the persistent excitation condition is met. Suppose that a reference trajectory is generated by an ideal oscillator and that the control objective is to make trajectory of real gyroscopes follow that of the reference model. The reference model is defined as

$$
\ddot{q}_{m}+K_{m} q_{m}=0
$$


where $K_{m}=\operatorname{diag}\left\{\omega_{1}^{2}, \omega_{2}^{2}\right\}$ are the reference resonant modes of both axis. We present following two theorems whose proof may be found in [10].

\section{Theorem 1 (Stability)}

With following control law (7) and parameter adaptation laws (8), the trajectory error $e_{p}=q-q_{m}$, and its time derivative $\dot{e}_{p}$ and $\ddot{e}_{p}$ converge globally and exponentially to zero.

$$
\begin{aligned}
& \tau=\hat{D} \dot{q}_{m}+\hat{R} q_{m}+2 \hat{\Omega} \dot{q}_{m}+\tau_{0} \\
& \dot{\hat{R}}=\frac{1}{2} \gamma_{R}\left(\tau_{0} q_{m}^{T}+q_{m} \tau_{0}^{T}\right) \\
& \dot{\hat{D}}=\frac{1}{2} \gamma_{D}\left(\tau_{0} \dot{q}_{m}^{T}+\dot{q}_{m} \tau_{0}^{T}\right) \\
& \dot{\hat{\Omega}}=\gamma_{\Omega}\left(\tau_{0} \dot{q}_{m}^{T}-\dot{q}_{m} \tau_{0}^{T}\right)
\end{aligned}
$$

where $R=K-K_{m}, \hat{D}, \hat{R}, \hat{\Omega}$ are estimates of $D, R$ and $\Omega, \tau_{0}=-\gamma \dot{e}_{p}$ and $\gamma=\operatorname{diag}\left\{\gamma_{1}, \gamma_{2}\right\}$

\section{Theorem 2 (Persistent excitation condition)}

With control law (7) and parameter adaptation laws (8), if the gyroscope is controlled to follow the mode-unmatched reference model, i.e. $\omega_{1} \neq \omega_{2}$, the persistent excitation condition is satisfied and all unknown gyroscope parameters, including the angular rate, are estimated correctly. 
Theorems 1 and 2 show that the motion of a mode-unmatched gyroscope, in which the resonance frequency of the $x$-axis is different from that of the $y$-axis, has sufficient persistence of excitation to permit the identification of all major fabrication imperfections as well as "input" angular rate. This means that adaptive controlled gyroscope has no ZRO and is self-calibrating.

\section{B. Velocity Observer-Based Adaptive Control}

The position and velocity measurements are corrupted by electrical noise in the sensing circuit. The analysis of the stochastic properties of the sensing noises, as well as the estimation of their intensity is given in literatures [5,11], and only results are presented here. The estimated power spectral densities of the position $\left(S_{p}\right)$ and velocity $\left(S_{v}\right)$ measurements is given by

$$
S_{p}=\left(\frac{2 C_{0}+C_{p}}{2 V_{0} \frac{d C}{d y}}\right)^{2} 4 k_{B} T R_{\text {wire }}, \quad S_{v}=\left(V_{D C} \frac{d C}{d y}\right)^{-2} \frac{k_{B} T}{R_{a m p}}
$$

Both are assumed zero-mean white noises. Ideally, the power spectral density of velocity measurement noise should be given by

$$
S_{v}=\omega^{2} S_{p}
$$

where $\omega$ is a resonant frequency of the gyroscope. However, current velocity sensing circuitry technology produces a noise with spectral power that is $3 \sim 4$ orders of magnitude larger than this ideal value. Thus, it is necessary to introduce an adaptive observer, to avoid measuring directly the velocity of the proof mass. 
In designing such a velocity observer, if we are careful not to modify the velocity measurement-based adaptive control structure, the analytic convergence and resolution results of the velocity measurement-based adaptive control can be easily extended for the case when velocity estimation is utilized. In order to estimate velocity, we introduce the following observer.

$$
\begin{aligned}
& \dot{\hat{q}}_{p}=\hat{q}_{v}+L\left(q-\hat{q}_{p}\right) \\
& \dot{\hat{q}}_{v}=-K_{m} \hat{q}_{p}
\end{aligned}
$$

where $\hat{q}_{p}$ is the estimate of the position, $\dot{\hat{q}}_{p}$ is the estimate of the velocity, $\hat{q}_{v}$ is an additional state of the velocity observer, and $L$ is a observer gain matrix given by $L=\operatorname{diag}\left\{L_{1}, L_{2}\right\}$. To complete the modification, the velocity term $\dot{q}$ in the adaptive control law given by (7) and parameter adaptation laws in (8) is replaced by $\dot{\hat{q}}_{p}$. In order to derive the closed loop error equations, we need to define the trajectory estimation error $\tilde{q}_{p}=\hat{q}_{p}-q$ and $\tilde{q}_{v}=\hat{q}_{v}-\dot{q}$. When the velocity term $\dot{q}$ in the adaptive control and parameter adaptation laws is replaced by the observer generated estimate $\dot{\hat{q}}_{p}$, the trajectory error, trajectory estimation error and parameter estimation error dynamics are given by the sum of a known linear time-varying and an unknown linear time-invariant components as follows:

$$
\dot{x}_{o}=A_{o}(t) x_{0}+A_{u} x_{o}+G_{0} w_{0}
$$

where $x_{o}=\left[\begin{array}{lllll}e_{p} & \dot{e}_{p} & \tilde{q}_{p} & \tilde{q}_{v} & \tilde{\theta}\end{array}\right]^{T}, w_{o}=\left[\begin{array}{ll}b & n\end{array}\right]^{T}$ and the known time varying term $A_{o}(t)$ is given by 


$$
A_{o}(t)=\left[\begin{array}{ccccc}
0 & I & 0 & 0 & 0 \\
-K_{m} & -\gamma & \gamma L & -\gamma & W^{T}\left(q_{m}, \dot{q}_{m}\right) \\
0 & 0 & -L & I & 0 \\
0 & \gamma & -K_{m}-\gamma L & \gamma & -W^{T}\left(q_{m}, \dot{q}_{m}\right) \\
0 & -\Gamma W\left(q_{m}, \dot{q}_{m}\right) \gamma & \Gamma W\left(q_{m}, \dot{q}_{m}\right) \gamma L & -\Gamma W\left(q_{m}, \dot{q}_{m}\right) \gamma & 0
\end{array}\right]
$$

the known noise distribution matrix is given by

$$
G_{o}=\left[\begin{array}{cc}
0 & 0 \\
I & -\gamma L \\
0 & L \\
-I & \gamma L \\
0 & -\Gamma W\left(q_{m}, \dot{q}_{m}\right) \gamma L
\end{array}\right]
$$

and the unknown time invariant term $A_{u}$ is

$$
A_{u}=\left[\begin{array}{ccccc}
0 & 0 & 0 & 0 & 0 \\
-R & -(D+2 \Omega) & 0 & 0 & 0 \\
0 & 0 & 0 & 0 & 0 \\
R & (D+2 \Omega) & 0 & 0 & 0 \\
0 & 0 & 0 & 0 & 0
\end{array}\right]
$$

where $b$ and $n$ are Brownian and position measurement noise, $W\left(q_{m}, \dot{q}_{m}\right)$ is signal regressor, $\tilde{\theta}=\hat{\theta}-\theta$ is parameter estimation errors and

$$
\begin{aligned}
& \Gamma=\operatorname{diag}\left\{\gamma_{R}, \frac{1}{2} \gamma_{R}, \gamma_{R}, \gamma_{D}, \frac{1}{2} \gamma_{D}, \gamma_{D}, \frac{1}{2} \gamma_{\Omega}\right\} \\
& W^{T}\left(q_{m}, \dot{q}_{m}\right)=\left[\begin{array}{ccccccc}
x_{m} & y_{m} & 0 & \dot{x}_{m} & \dot{y}_{m} & 0 & -2 \dot{y}_{m} \\
0 & x_{m} & y_{m} & 0 & \dot{x}_{m} & \dot{y}_{m} & 2 \dot{x}_{m}
\end{array}\right] \\
& \theta^{T}=\left[\begin{array}{lllllll}
r_{x x} & r_{x y} & r_{y y} & d_{x x} & d_{x y} & d_{y y} & \Omega_{z}
\end{array}\right]
\end{aligned}
$$

where $r_{i j}, d_{i j}$ and $\Omega_{z}$ are respectively elements of $R, D$ and $\Omega$. 
The mean trajectory of the system under the stochastic environment is the same as the deterministic case and its convergence properties are also the same as the deterministic case. Unfortunately, the trajectory and trajectory estimation error dynamics part of (12) are not strictly positive real (SPR), and therefore it is difficult to prove the stability of this system using standard adaptive control techniques, based on the use of a Lyapunov function candidate. In order to prove stability, we will make use of the fact that $A_{o}(t)$ is a periodic time-varying matrix with known period $T=4 \pi^{2} /\left(\omega_{1} \omega_{2}\right)$, where $\omega_{1}$ and $\omega_{2}$ are model reference frequencies. The stability of periodic time-varying linear systems can be analyzed using Floquet-Lyapunov theory [12].

\section{Theorem 3 (Stability)}

Given the observer (11), the adaptive control and parameter adaptation laws, it is always possible to choose a velocity observer gain $L$, which makes the closed loop error dynamics (12) locally, uniformly and exponentially stable.

\section{Proof:}

According to Floquet-Lyapunov theory, there exists a periodic transformation matrix that converts a periodic time-varying linear system into a time invariant linear system [12]. Let $\Phi(t, 0)$ be a state transition matrix of the known linear part of (12), i.e.,

$$
\frac{d \Phi(t, 0)}{d t}=A_{o}(t) \Phi(t, 0)
$$

then it can be written as product of two matrices as 


$$
\Phi(t, 0)=F(t) \exp (\bar{A} t)
$$

where $F(t)$ is a continuous periodic nonsingular matrix with period $T$, which satisfies the condition $F(0)=F(T)=I$ and $\bar{A}$ is a constant matrix. The stability of a linear known part is determined by the eigenvalues of $\bar{A}$. In order to determine $F(t)$, the state transition matrix $\Phi(t, 0)$ must be computed. However, there is no simple way to compute $\Phi(t, 0)$ analytically. Instead, the transition matrix at the end of one period is computed by numerical integration of Eq. (13) and $\bar{A}$ is obtained by

$$
\bar{A}=\frac{1}{T} \ln (\Phi(T, 0))
$$

where $\exp (\ln (\Phi(T, 0))=\Phi(T, 0)$. In Fig. 2, the calculated stability boundaries of the time varying part of Eq. (12) are presented in terms of the observer gain $L$, for various reference model frequencies. As shown in the figure, it is always possible to choose a velocity observer gain $L$ such that $\bar{A}$ is asymptotically stable. Now, let the Lyapunov candidate be

$$
V=x_{o}^{T}(t) F^{-T}(t) M F^{-1}(t) x_{o}(t)
$$

where $M$ is the solution of the Lyapunov function, $M \bar{A}+\bar{A}^{T} M=-I$. Since $\bar{A}$ is asymptotically stable, $M>0$ and $F^{-T}(t) M F^{-1}(t)>0$ for all $t \geq 0$. Differentiating $V$ with respect to time, we obtain

$$
\dot{V}=-x_{o}^{T}\left(F^{-T} F^{-1}\right) x_{o}+2 x_{o}^{T} F^{-T} M F^{-1} A_{u}
$$

Since $F(t)$ is a nonsingular matrix for all $t \geq 0, F^{-T}(t) F^{-1}(t)>0$ for all $t \geq 0$. Thus,

$$
\begin{aligned}
\dot{V} & \leq-\left(\alpha_{\min }^{2}-2 \alpha_{\max }^{2} \beta \lambda_{\text {max }}(M)\right)\left\|x_{o}\right\|^{2} \\
& <0
\end{aligned}
$$


within the domain of attraction,

$$
\alpha_{\min }^{2}>2 \alpha_{\max }^{2} \beta \lambda_{\text {max }}(M)
$$

where $\alpha_{\min }=\min _{0 \leq t \leq T}\left\|F^{-1}(t)\right\|, \alpha_{\max }=\max _{0 \leq t \leq T}\left\|F^{-1}(t)\right\|$ and $\beta=\left\|A_{u}\right\|$. Notice that the unknown matrix $A_{u}$ is composed of the damping $D$, frequency modeling error $R$ and applied angular rate $\Omega$, which all have very small values. Therefore, $\beta$ is a small number.

\section{PERFormance ANALYSiS}

We now examine the convergence rate and stochastic variance of the angular rate estimate. This analysis gives us an estimate of the bandwidth and resolution of an adaptive controlled gyroscope.

\section{A. Convergence Rate Analysis}

In this section, the parameter convergence rate of the adaptive control scheme designed in previous section is studied using averaging analysis. Averaging analysis is commonly used in the adaptive control literature [13], and will be used to estimate the convergence properties of gyroscope parameter estimates including the applied angular rate. The convergence rate of the angular rate estimate is important because it determines the bandwidth of the gyroscope.

Using the fact that parameter estimation dynamics is slower than trajectory and trajectory estimation dynamics, we can relate the slow parameter estimation dynamics with the following averaged dynamics. 


$$
\dot{\tilde{\theta}}_{a v}=-\Gamma A V G\left\{W\left(q_{m}, \dot{q}_{m}\right) \hat{M}_{o}\left(W^{T}\left(q_{m}, \dot{q}_{m}\right)\right)\right\} \tilde{\theta}_{a v}
$$

where $\hat{M}_{o}$ is a transfer function matrix,

$$
\hat{M}_{o}(s)=\left[\begin{array}{cc}
\hat{M}_{o 1}(s) & 0 \\
0 & \hat{M}_{o 2}(s)
\end{array}\right]
$$

where

$$
\begin{aligned}
& \hat{M}_{o 1}(s)=\frac{\gamma_{1} L_{1} s^{2}}{s^{4}+L_{1} s^{3}+\left(2 \omega_{1}^{2}+\gamma_{1} L_{1}\right) s^{2}+\omega_{1}^{2} L_{1} s+\omega_{1}^{4}} \\
& \hat{M}_{o 2}(s)=\frac{\gamma_{2} L_{2} s^{2}}{s^{4}+L_{2} s^{3}+\left(2 \omega_{2}^{2}+\gamma_{2} L_{2}\right) s^{2}+\omega_{2}^{2} L_{2} s+\omega_{2}^{4}}
\end{aligned}
$$

Note that the transfer function $\hat{M}_{o}(s)$ has two different forcing frequencies, i.e., one is the $x$-axis resonant frequency and the other is the $y$-axis resonant frequency, i.e.

$$
\begin{array}{ll}
x_{m}=X_{0} \sin \left(\omega_{1} t\right), & \dot{x}_{m}=X_{0} \omega_{1} \cos \left(\omega_{1} t\right) \\
y_{m}=Y_{0} \sin \left(\omega_{2} t\right), & \dot{y}_{m}=Y_{0} \omega_{2} \cos \left(\omega_{2} t\right)
\end{array}
$$

Therefore, the filtered steady-state response through $\hat{M}_{o}\left(W^{T}\left(q_{m}, \dot{q}_{m}\right)\right)$ is

$$
\hat{M}_{o}\left(W^{T}\right)=\left[\begin{array}{cc}
X_{0} \sin \left(\omega_{1} t\right) & 0 \\
A_{1} Y_{0} \sin \left(\omega_{2} t+\phi_{1}\right) & A_{2} X_{0} \sin \left(\omega_{1} t+\phi_{2}\right) \\
0 & Y_{0} \sin \left(\omega_{2} t\right) \\
X_{0} \omega_{1} \cos \left(\omega_{1} t\right) & 0 \\
A_{1} Y_{0} \omega_{2} \cos \left(\omega_{2} t+\phi_{1}\right) & A_{2} X_{0} \omega_{1} \cos \left(\omega_{1} t+\phi_{2}\right) \\
0 & Y_{0} \omega_{2} \cos \left(\omega_{2} t\right) \\
-2 A_{1} Y_{0} \omega_{2} \cos \left(\omega_{2} t+\phi_{1}\right) & 2 A_{2} X_{0} \omega_{1} \cos \left(\omega_{1} t+\phi_{2}\right)
\end{array}\right]^{T}
$$

where 


$$
\begin{aligned}
& A_{1}=\frac{\gamma_{1} L_{1} \omega_{2}^{2}}{\sqrt{\left(\gamma_{1} L_{1} \omega_{2}^{2}-\left(\omega_{1}^{2}-\omega_{2}^{2}\right)^{2}\right)^{2}+L_{1}^{2} \omega_{2}^{2}\left(\left(\omega_{1}^{2}-\omega_{2}^{2}\right)^{2}\right.}} \\
& \phi_{1}=\tan ^{-1} \frac{L_{1} \omega_{2}\left(\omega_{1}^{2}-\omega_{2}^{2}\right)}{\gamma_{1} L_{1} \omega_{2}^{2}-\left(\omega_{1}^{2}-\omega_{2}^{2}\right)^{2}} \\
& A_{2}=\frac{\gamma_{2} L_{2} \omega_{1}^{2}}{\sqrt{\left(\gamma_{2} L_{2} \omega_{1}^{2}-\left(\omega_{1}^{2}-\omega_{2}^{2}\right)^{2}\right)^{2}+L_{2}^{2} \omega_{1}^{2}\left(\left(\omega_{1}^{2}-\omega_{2}^{2}\right)^{2}\right.}} \\
& \phi_{2}=\tan ^{-1} \frac{-L_{2} \omega_{1}\left(\omega_{1}^{2}-\omega_{2}^{2}\right)}{\gamma_{2} L_{2} \omega_{1}^{2}-\left(\omega_{1}^{2}-\omega_{2}^{2}\right)^{2}}
\end{aligned}
$$

A sufficient condition for $\tilde{\theta}_{a v}$ in Eq. (18) to converge to zero is that cross-correlation matrix $R_{w \hat{w}}=A V G\left(W \hat{M}_{o}\left(W^{T}\right)\right)$ is a positive-definite. If the gains $\gamma_{1}$ and $\gamma_{2}$ are too small, and/or $\omega_{1}$ and $\omega_{2}$ are too far apart, the magnitudes $A_{1}$ and $A_{2}$ are negligible and phase $\phi_{1,2} \geq\left| \pm 90^{\circ}\right|$. In that case, the filtered steady-state response of the cross-axis signals, including the angular rate term, cannot make any significant contribution to the crosscorrelation matrix $R_{w \hat{w}}$ or may cause instability. This results in large un-damped oscillations, or divergence in the parameter estimation response. On the other hand, when $\omega_{1}$ and $\omega_{2}$ are too close to each other, the error dynamics response still results in large undamped oscillations, because of lack of persistence of excitation. It is important to mention here that the observer gain $L$ should be chosen such that the closed loop system is stable, which is always possible to do. The appropriate choice for the frequency ratio $\Delta \omega=\omega_{2} / \omega_{1}$ also depends on the choice of the control gains $\gamma_{1}$ and $\gamma_{2}$. Selecting gains $\gamma_{1}$ and $\gamma_{2}$ to be too small, makes the choice of an appropriate $\Delta \omega=\omega_{2} / \omega_{1}$ hard, since a 
slight mismatch in $\omega_{1}$ and $\omega_{2}$ results in small values for $A_{1,2}$ and $\left|\phi_{1,2}\right|>90^{\circ}$. Selecting large values for observer gains makes the response of the gyroscope resemble that of the velocity measurement-based gyroscope. According to the simulation study that will be subsequently described in this section, a ratio between $10 \%$ to $40 \%$ between the two resonant frequencies is a reasonable choice, when sufficiently large values of $\gamma_{1}$ and $\gamma_{2}$, and appropriate values of gains $L_{1}$ and $L_{2}$ are employed.

Using the facts that the products of sinusoids at different frequencies have zero average, the average equation for parameter estimate error dynamics can be obtained. All cross-term parameter estimates dynamics are coupled with each other. However, as the control gains $\gamma_{1}$ and $\gamma_{2}$, and observer gains $L_{1}$ and $L_{2}$ are made sufficiently large and/or the reference model resonant frequencies $\omega_{1}$ and $\omega_{2}$ are close enough, all cross-terms in the parameter estimates dynamics become less coupled, because $A_{1} \approx A_{2} \approx 1$ and $\phi_{1} \approx \phi_{2} \approx 0$. In this case, the parameter estimates errors are almost uncoupled with each other, except for the estimates errors of the asymmetric damping term and the angular rate. Their dynamics are coupled and given by

$$
\left[\begin{array}{c}
\dot{\tilde{d}}_{\text {xyav }} \\
\dot{\tilde{\Omega}}_{z a v}
\end{array}\right] \approx\left[\begin{array}{ll}
a_{11} & a_{12} \\
a_{21} & a_{22}
\end{array}\right]\left[\begin{array}{l}
\tilde{d}_{x y a v} \\
\tilde{\Omega}_{z a v}
\end{array}\right]
$$

where

$$
\begin{aligned}
& a_{11}=-\frac{\gamma_{D}}{4}\left(X_{0}^{2} \omega_{1}^{2}+Y_{0}^{2} \omega_{2}^{2}\right), a_{12}=-\frac{\gamma_{D}}{2}\left(X_{0}^{2} \omega_{1}^{2}-Y_{0}^{2} \omega_{2}^{2}\right) \\
& a_{21}=-\frac{\gamma_{\Omega}}{2}\left(X_{0}^{2} \omega_{1}^{2}-Y_{0}^{2} \omega_{2}^{2}\right), a_{22}=-\gamma_{\Omega}\left(X_{0}^{2} \omega_{1}^{2}+Y_{0}^{2} \omega_{2}^{2}\right)
\end{aligned}
$$


By the Eq. (20), if we set the reference model oscillations such that $X_{0} \omega_{1}=Y_{0} \omega_{2}$, the dynamics of the angular rate estimate can be decoupled from that of the asymmetric damping estimate. In this case, all estimates dynamics is almost decoupled, and therefore it is possible to adjust the dynamics of angular rate estimate independently, without significantly affecting the estimation dynamics of fabrication imperfections.

Applying this decoupling condition, the average dynamics of the angular rate estimate is approximately given by

$$
\dot{\widetilde{\Omega}}_{z a v} \approx-2 \gamma_{\Omega} X_{0}^{2} \omega_{1}^{2} \widetilde{\Omega}_{z a v}
$$

This is the exactly same result that is obtained for the adaptive control system without velocity estimation [14]. Thus, the bandwidth of the adaptive controlled gyroscope with velocity estimation is also approximately given by $B W \approx 2 \gamma_{\Omega} X_{0}^{2} \omega_{1}^{2}$, which implies that the bandwidth of the MEMS gyroscope under the observer based adaptive control is proportional to the adaptation gain $\gamma_{\Omega}$ and the energy of oscillation of the reference model. Figure 3 and 4 show the comparison between analytical convergence rate of angular rate given by Eq. (21) and the simulation results for various resonant frequency ratios and control gains. The observer-based adaptive control system derived is more sensitive to the variations in the resonant frequency ratio and control gains than the velocity measurementbased adaptive control design. This is because, given a moderate value for the observer gains, the phase differences in $\hat{M}_{o}(s)$ are larger than that of the adaptive control case for the same changes in resonant frequency. Although large control gains $\gamma_{1}$ and $\gamma_{2}$ are good for decoupling the parameter estimation dynamics, selecting large values for these control 
gains is not desirable since they may cause large overshoot in the transient response of the gyroscope dynamics and may cause decrease the resolution performance of the gyroscope, as well be discussed in the next sub-section. Figure 5 shows a comparison between the analytical convergence rate of the angular rate estimate given by Eq. (21) and simulation results for various observer gains. As shown in Fig. 5, if the observer gain is sufficiently large, the actual convergence rate is very close to the analytical result.

\section{B. Resolution Analysis}

Measurement and Brownian noises limit the minimum detectable signal of angular rate estimate. Brownian noise is a thermal noise that is produced by the collisions between air molecules and the structure, or by viscoelastic effects in the suspension of the gyroscope, and enters to the system as a noisy force generator. Brownian noise can be modeled as a zero-mean white input noise, and its power spectral density is given by $S_{b}=4 k_{B} T d / \mathrm{m}^{2}$ [4], where $m$ is the mass of the proof mass and $d$ is a damping coefficient. The standard deviation of the angular rate estimate error, or resolution, is obtained from covariance matrix of $x_{o}$ of Eq. (12). Covariance $P_{o}$ of $x_{o}$ can be easily pre-computed independently with mean trajectory by solving the following familiar Lyapunov equation.

$$
\dot{P}_{o} \approx P_{o} A_{o}^{T}+A_{o} P_{o}+G_{o} S_{o} G_{o}^{T}
$$

where $S_{o}=\operatorname{diag}\left\{S_{b}, S_{p}\right\}$. Resolution of angular rate estimate, $\sigma_{\Omega}$, is computed by

$$
\sigma_{\Omega}=\sqrt{C P_{o} C^{T}}
$$


where $C=\left[0_{1 \times 14} 1\right]$. The ultimate achievable resolution can be calculated by setting $S_{p}=0$ and computing $\sigma_{\Omega}$ using Eq. (23).

Figure 6 shows the effects of various design parameters such as control gains and parameter adaptation gains on the variance of the angular rate estimate error. The plots in Fig. 6 were obtained from the time domain response of Eq. (23) and the steady-state values represent the resulting steady-state covariance. Except for the fact that control gain variations make slight changes in the covariance matrix $P_{o}$, only the angular rate adaptation gain $\gamma_{\Omega}$ significantly affects the variance. This implies that the resolution can be adjusted with the angular rate adaptation gain independently, without significantly affecting the other dynamics of the fabrication imperfection estimates. The resolution performance of the observer-based adaptive controlled gyroscope is almost the same as the one that would be obtained if the power spectral density of velocity measurement noise is ideally given by Eq. (10).

\section{Advantages of Adaptive Mode of Operation}

The main advantages of the adaptive mode of operation, proposed in this paper, include self-calibration, large robustness to parameter variations, and no zero-rate output. Moreover, because a single adaptive scheme controls all operation tasks of the gyroscope, i.e. from initiating the vibratory motion of proof mass to estimating the angular rate, analytic predictions for the bandwidth and resolution of the gyroscope are easy to obtain and relatively precise. The proposed adaptive controller design is also easy to implement in high $Q$ systems. Thus, the noise properties associated with a high $Q$ system can be fully 
utilized. Another advantage of the adaptive mode of operation is that it is easy to adjust the trade-off between bandwidth and resolution by simply adjusting the angular rate adaptation gain. In contrast, in a gyroscope operating under the conventional open-loop or forcebalancing closed-loop mode of operation, the bandwidth and ultimate resolution of the gyroscope depend on the low-pass filter characteristics that is used to demodulate the angular rate estimate. Thus, it is difficult to adjust both bandwidth and resolution, without changing the demodulation filter. Therefore, the adaptive mode of operation is better suited for medium-cost gyroscopes that are used in high-performance applications. One disadvantage of the adaptive mode of operation is that it cannot be applied to a conventional gyroscope structure, since it requires the unmatched resonance mode of the gyroscopes and equal movements in the $x$ and $y$ axes. This means that for applying proposed adaptive operation scheme of MEMS gyroscopes, new gyroscope should be designed so that equal movements in the $x$ and $y$ axes allow. Figure 7 shows a comparison between a conventional mode and an adaptive mode of operation. Detailed description of the design and fabrication process of new MEMS gyroscope is in reference [10].

\section{Simulations}

A simulation study using the preliminary design data of the MIT-SOI MEMS gyroscope was conducted, to test the analytical results presented in this paper and verify its predicted performance. The data of some of gyroscope parameters in the model is summarized in Table 2. For simulation purposes, we allowed $\pm 5 \%$ parameter variations for the spring and 
damping coefficients and assumed $\pm 1 \%$ magnitude of nominal spring and damping coefficients for their off-diagonal terms. Notice that the simulation results are shown in non-dimensional units, which are non-dimensionalized based on the proof-mass, length of one micron and $x$-axis nominal natural frequency.

Figure 8 shows the time responses of the estimation errors fr the various gyroscope parameters. The estimate of angular rate response to step input angular rate is shown in Fig. 9. In this figure, the upper and lower bounds of its analytically estimated standard deviation are also plotted. Figure 10 shows the estimate of angular rate response to sinusoidal input angular rate. These simulation results support the theoretical results obtained in this paper.

\section{CONCLUSIONS}

Dynamic analysis of typical MEMS gyroscopes shows that fabrication imperfections are a major factor limiting the performance of the gyroscope. Thus, the main purpose of gyroscope control should be to null out these imperfections and cross-couplings effectively during the operation of the gyroscope. However, the motion of a conventional modematched z-axis gyroscope does not have sufficient persistence of excitation and, as a result, all major fabrication imperfections cannot be identified and compensated for in an on-line fashion. Moreover, some types of fabrication imperfections, which can be modeled as cross-damping terms, produce inherent zero-rate output (ZRO).

An analysis technique for identifying z-axis gyroscope operating conditions, which permit the on-line compensation of fabrication imperfections and self-calibration, was developed. 
It showed that the motion of a mode-unmatched gyroscope, in which the resonance frequency of the $x$-axis is different from that of the $y$-axis, has sufficient persistence of excitation to permit the identification of all major fabrication imperfections as well as "input" angular rate. Based on this analysis, new operation strategies were formulated for MEMS gyroscopes with two un-matched oscillatory modes. A new adaptive control algorithm with velocity estimation was developed, which operates with only measurements of the $x$ and $y$ positions of the proof mass. The parameter adaptation algorithm (PAA) in the adaptive controller simultaneously estimates the component of the angular velocity vector, which is orthogonal to the plane of oscillation of the gyroscope (the $z$-axis) and the linear damping and stiffness model coefficients. The convergence and resolution analysis presented in paper showed that the proposed adaptive controlled scheme offers several advantages over conventional modes of operation. These advantages include a larger operational bandwidth, absence of zero-rate output, self-calibration and a large robustness to parameter variations, which are caused by fabrication defects and ambient conditions.

A simulation study using the preliminary design data of the MIT-SOI MEMS gyroscope was conducted, to test the analytical results derived in this paper and to verify the predicted performance of the different proposed controlled schemes. Simulation results were in strong agreement with the analytically derived predicted results and performance estimates.

\section{ACKNOWLEDGEMENTS}

This research was supported by DARPA under Contract N66001-97-C-8643. 


\section{NOMENCLATURE}

$A V G(\cdot)$ : AVERAGE OF (·)

${ }^{a} d \vec{b} / d t$ : time derivative of a vector $\vec{b}$ in the frame $\{a\}$

$C_{p}, C_{0}$ : parasite and nominal sensing capacitances

$d_{1}, d_{2}$ : damping coefficients

$I$ : identity matrix

$k_{1}, k_{2}:$ spring coefficients

$k_{B}$ : Boltzmann's constant

$m:$ proof mass

$Q_{x}, Q_{y}:$ quality factors of $x$ and $y$-axis

$R_{\text {wire }}, R_{\text {amp }}:$ wiring and amplifier resistances

$T:$ absolute temperature

$V_{0}, V_{D C}:$ nominal and DC voltages

$X_{0}, Y_{0}:$ amplitudes of $x$ and $y$-axis oscillation

$\Omega_{x}, \Omega_{y}, \Omega_{z}$ : angular velocity components along $x, y$ and $z$-axis of the gyro frame

$\tau_{x}, \tau_{y}:$ control forces along $x$ and $y$-axis of the gyro frame

$\lambda_{\max }(\cdot)$ : maximum eigenvalue of $(\cdot)$ 
$\|\cdot\|:$ norm of vector or matrix

$(\cdot)^{T}:$ transpose of $(\cdot)$

$(\cdot)^{-1}$ : inverse of $(\cdot)$

\section{REFERENCES}

[1] Yazdi, N., Ayazi, F., and Najafi, K., 1998, "Micromachined Inertial Sensors", Proceedings of the IEEE, Vol.86, No.8, pp.1640-1659.

[2] Shkel A., Howe, R. T., and Horowitz, R., 1999, "Modeling and simulation of micromachined gyroscopes in the presence of imperfection", Int. Conf. On Modelling and Simulation of Microsystems, Puerto Rico, U.S.A., pp. 605-608.

[3] Lawrence, A., 1993, Modern Inertial Technology: Navigation, Guidance and Control, Springer Verlag.

[4] Clark, W. A., 1997, Micromachined Vibratory Rate Gyroscopes, Ph.D. Thesis, U.C. Berkeley.

[5] Juneau, T. N., 1997, Micromachined Dual Input Axis Rate Gyroscope, Ph.D. Thesis, U.C. Berkeley.

[6] Loveday, P. W., and Rogers, C. A., 1998, "Modification of Piezoelectric Vibratory Gyroscope Resonator Parameters by Feedback ControP', IEEE Transactions on Ultrasonics, Ferroelectrics and Frequency Control, Vol.45, No.5, pp.1211-1215.

[7] Ljung, P. B., 1997, Micromachined Gyroscope with Integrated Electronics, Ph.D. Thesis, U.C. Berkeley. 
[8] Jiang, X., Seeger, J., Kraft, M., and Boser, B. E., 2000, “A monolithic surface micromachined Z-axis gyroscope with digital output", 2000 Symposium on VLSI Circuits, Honolulu, HI, USA, pp.16-19.

[9] Shkel, A. M., Horowitz, R., Seshia, A., Park, S., and Howe, R. T., 1999, "Dynamics and Control of Micromachined Gyroscopes", Proceedings of the American Control Conference, pp.2119-2124.

[10] Park, S., 2000, Adaptive Control Strategies for MEMS Gyroscopes, Ph.D. Thesis, U.C. Berkeley.

[11] Boser, B. E, 1997, "Electronics for Micromachined Inertial Sensors", International Conference on Solid-State Sensors and Actuators, pp.1169-1172.

[12] Chen, C-T, 1984, Linear System Theory and Design, CBS College Publishing.

[13] Sastry, S. S., 1989, Adaptive Control: Stability, Convergence and Robustness, Prentice Hall.

[14] Park, S., and Horowitz, R., 2000, “Adaptive Control of MEMS Gyroscopes”, The $7^{\text {th }}$ Mechatronics Forum International Conference, Atlanta, GA, USA. 
Figure 1. A model of a MEMS z-axis gyroscope

Figure 2. Stability bounds with respect to observer gain $L$ :

(a) $\gamma=I, \omega_{1}=1, \omega_{2}=\sqrt{0.5}$, (b) $\gamma=I, \omega_{1}=1, \omega_{2}=1$

(c) $\gamma=I, \omega_{1}=1, \omega_{2}=\sqrt{1.5}$, (d) $\gamma=I, \omega_{1}=1, \omega_{2}=\sqrt{2}$

(e) $\gamma=0.5 I, \omega_{1}=1, \omega_{2}=\sqrt{1.5}, \quad$ (f) is the same as (c)

Figure 3. Convergence rate comparisons between analytical equation and various ratios of resonant frequencies

Figure 4. Convergence rate comparisons between analytical equation and various control gains

Figure 5. Convergence rate comparisons between analytical equation and various observer gains

Figure 6. Variance variations of angular rate estimate error $\tilde{\Omega}_{z}$ due to

(a) angular rate adaptation gain $\gamma_{\Omega}$, (b) control gains $\gamma_{1,2}$,

(c) spring coefficient adaptation gain $\gamma_{R}$, (d) damping coefficient adaptation

Figure 7. Comparison between a conventional and an adaptive mode

Figure 8. Parameter estimation errors: (a) damping coefficients, (b) spring coefficients

Figure 9. Time responses of angular rate estimate to the $5 \mathrm{deg} / \mathrm{sec}$ step input

Figure 10. Time responses of angular rate estimate to the $5 \mathrm{deg} / \mathrm{sec}$ sinusoid input at $50 \mathrm{~Hz}$ 


\begin{tabular}{|c|c|}
\hline parameter & Value \\
\hline mass & $5.095 \times 10^{-7} \mathrm{~kg}$ \\
\hline x-axis frequency & $4.17 \mathrm{KHz}$ \\
\hline y-axis frequency & $5.11 \mathrm{KHz}$ \\
\hline Quality factor & $10^{4}$ \\
\hline Brownian noise PSD & $1.47 \times 10^{-26} \mathrm{~N}^{2} \mathrm{sec}$ \\
\hline Position noise PSD & $1.49 \times 10^{-27} \mathrm{~m}^{2} \mathrm{sec}$ \\
\hline Velocity noise PSD & $2.94 \times 10^{-12}(\mathrm{~m} / \mathrm{sec})^{2} \mathrm{sec}$ \\
\hline
\end{tabular}

Table 2. Key parameters of the gyroscope 


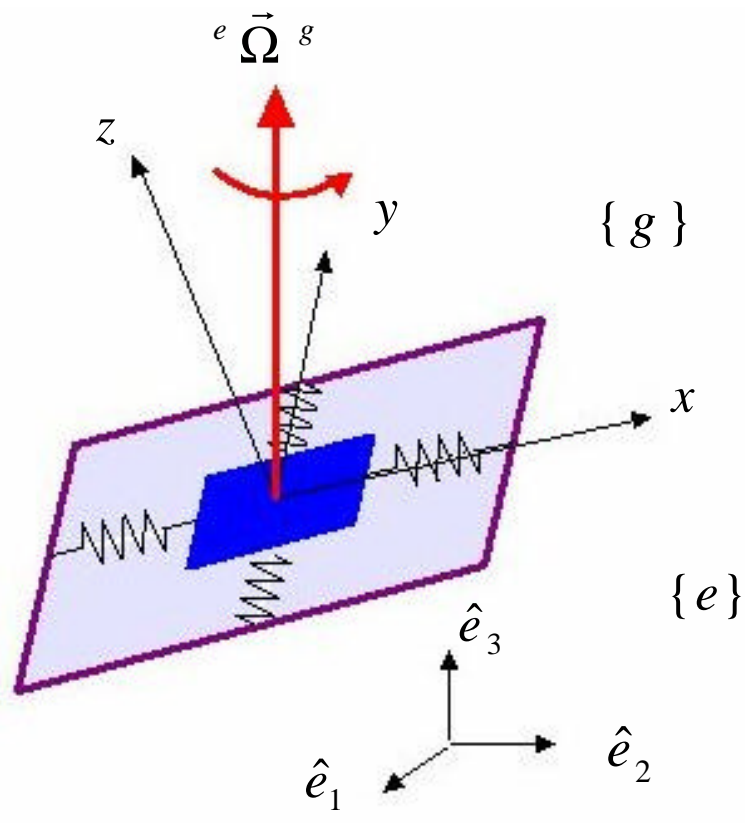

Figure 1. A model of a MEMS $z$ axis gyroscope 
(a)
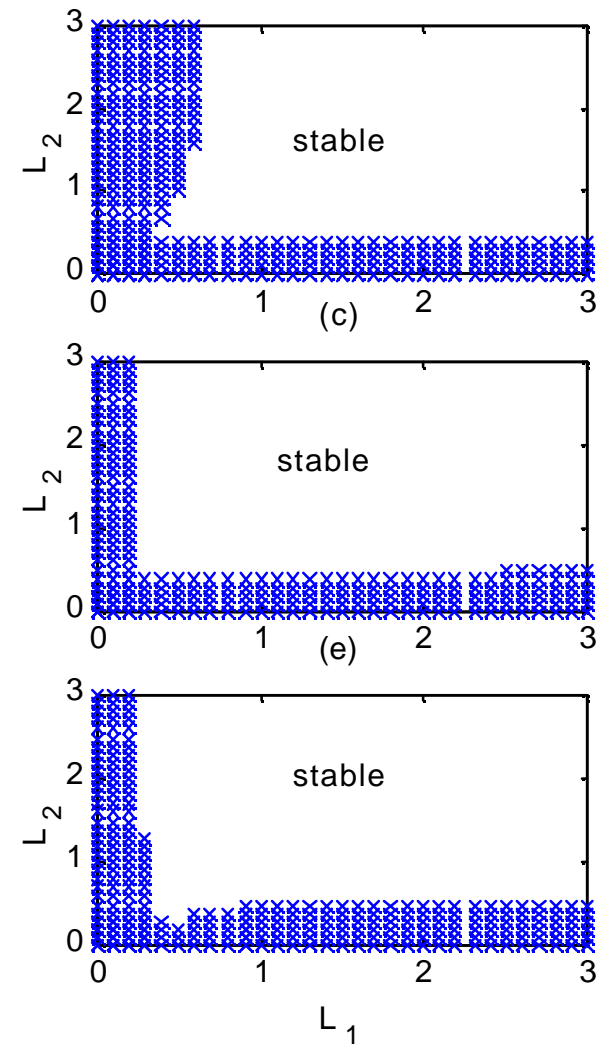

(b)
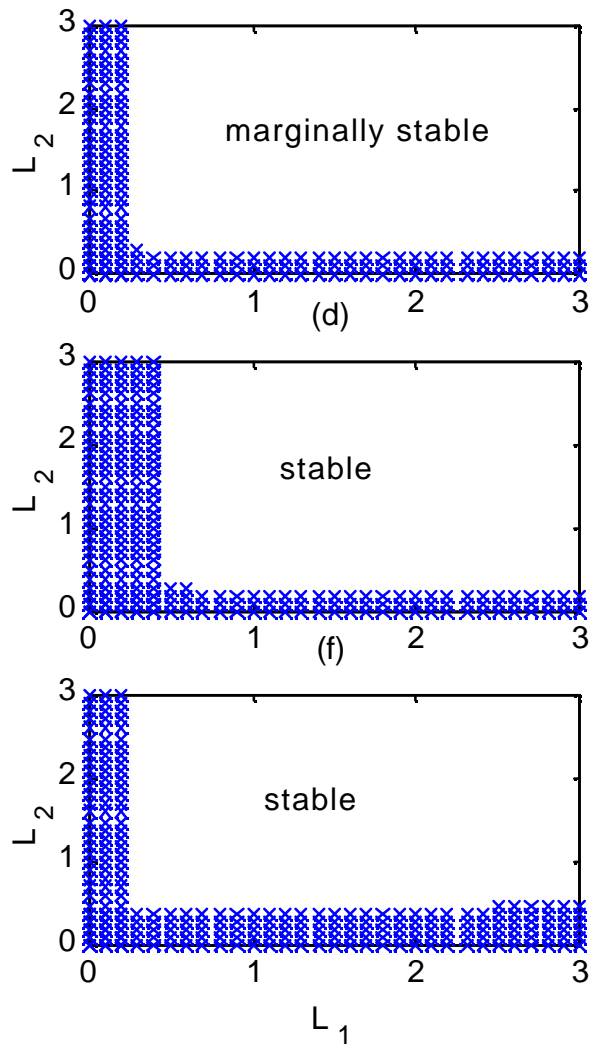

Figure 2. Stability bounds with respect to observer gain $L$ :

(a) $\gamma=I, \omega_{1}=1, \omega_{2}=\sqrt{0.5}$, (b) $\gamma=I, \omega_{1}=1, \omega_{2}=1$

(c) $\gamma=I, \omega_{1}=1, \omega_{2}=\sqrt{1.5}$, (d) $\gamma=I, \omega_{1}=1, \omega_{2}=\sqrt{2}$

(e) $\gamma=0.5 I, \omega_{1}=1, \omega_{2}=\sqrt{1.5}$, (f) is the same as (c) 


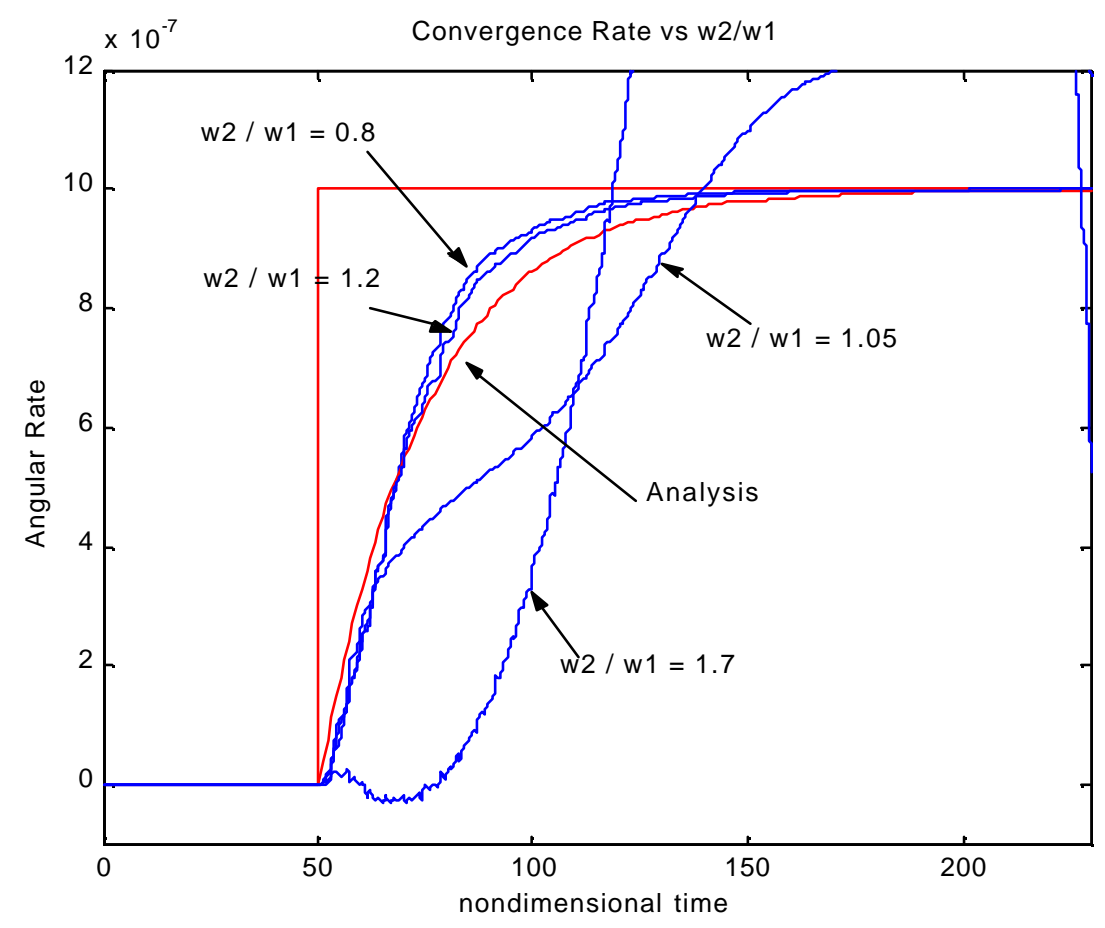

Figure 3. Convergence rate comparisons between analytical equation and various ratios of resonant frequencies 


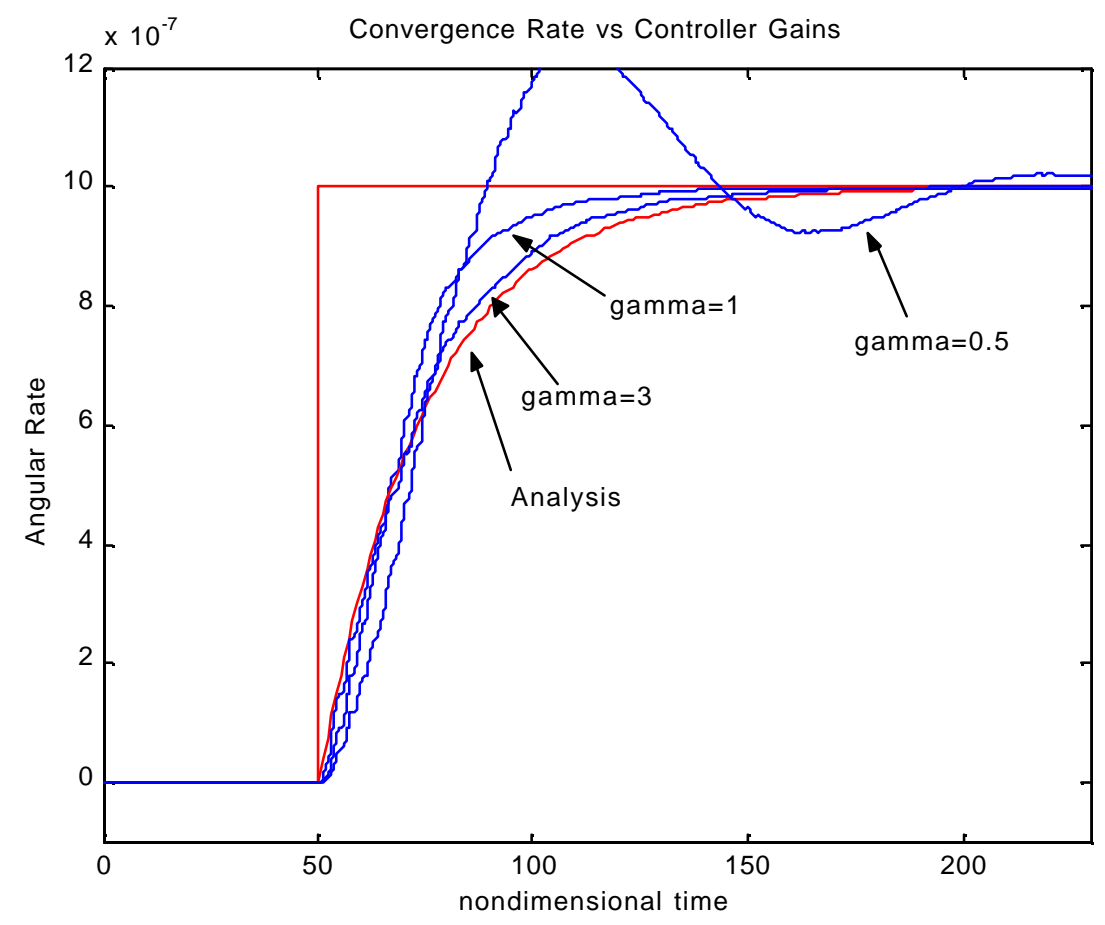

Figure 4. Convergence rate comparisons between analytical equation and various control gains 


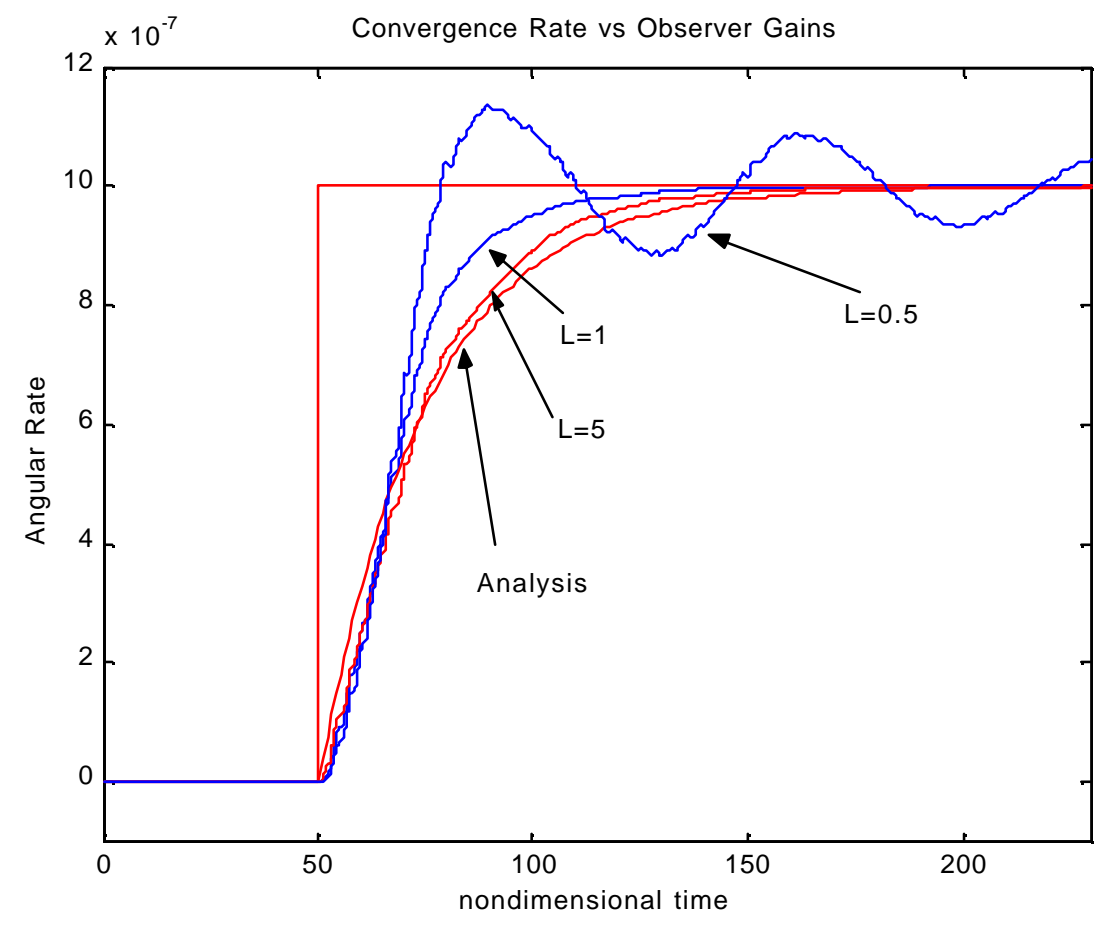

Figure 5. Convergence rate comparisons between analytical equation and various observer gains 

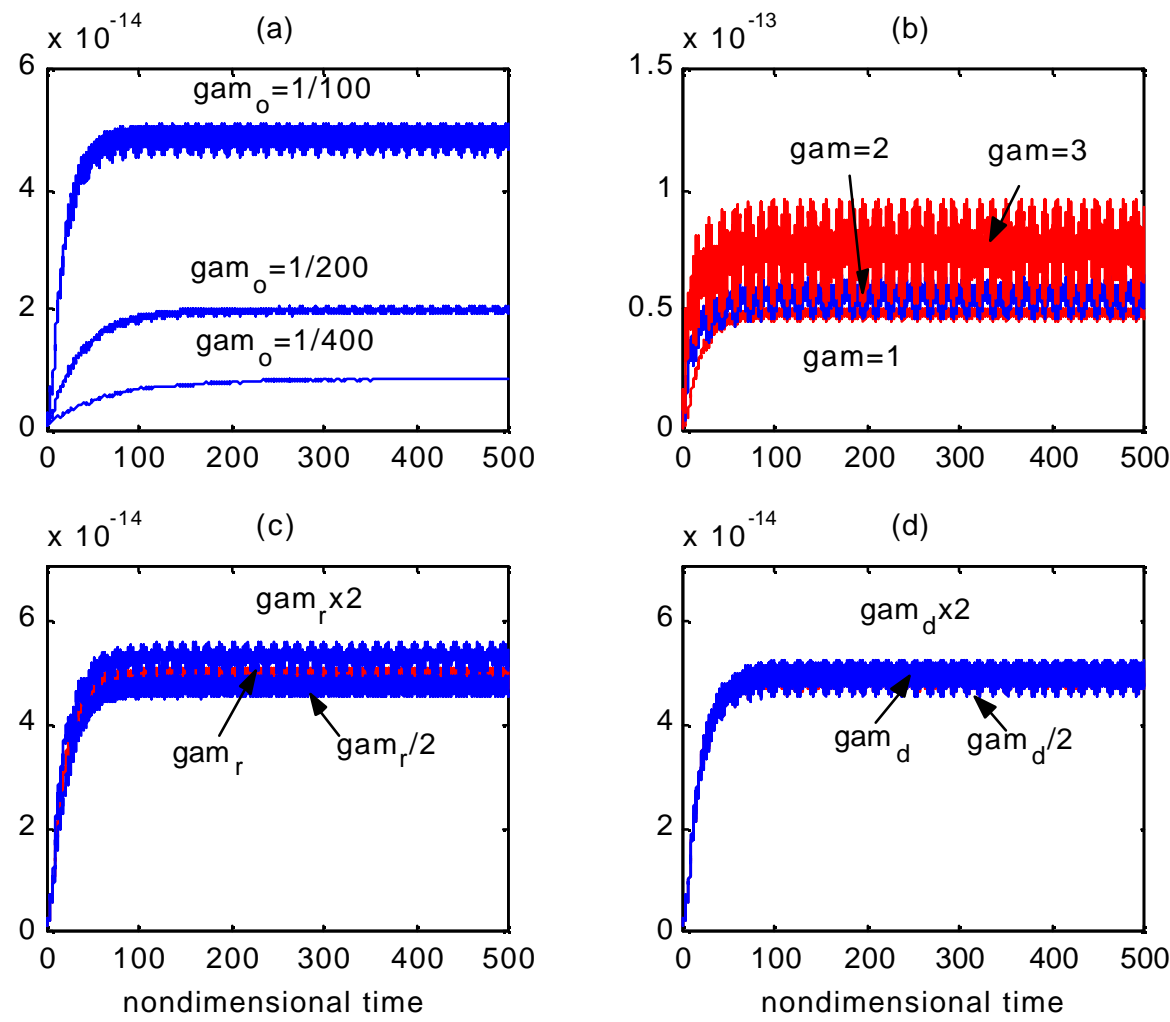

Figure 6. Variance variations of angular rate estimate error $\tilde{\Omega}_{z}$ due to

(a) angular rate adaptation gain $\gamma_{\Omega}$, (b) control gains $\gamma_{1,2}$,

(c) spring coefficient adaptation gain $\gamma_{R}$, (d) damping coefficient adaptation 


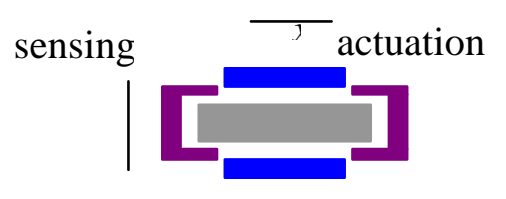

(a) conventional mode

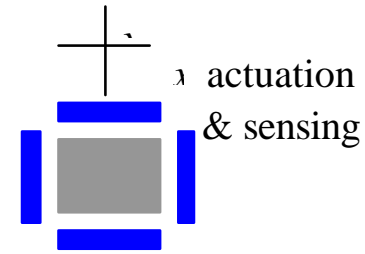

(b) adaptive mode

Figure 7. Comparison between a conventional and an adaptive mode 


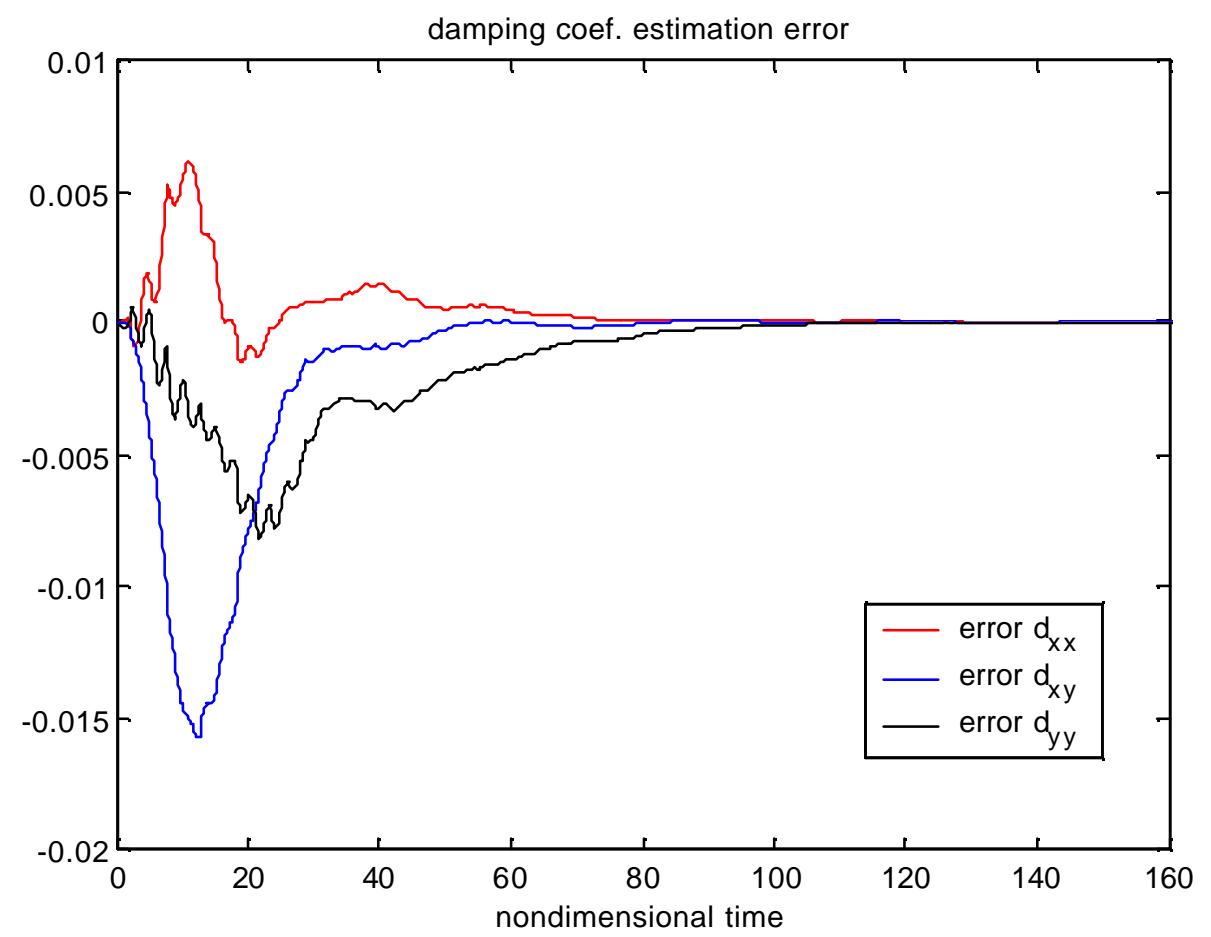

(a) 


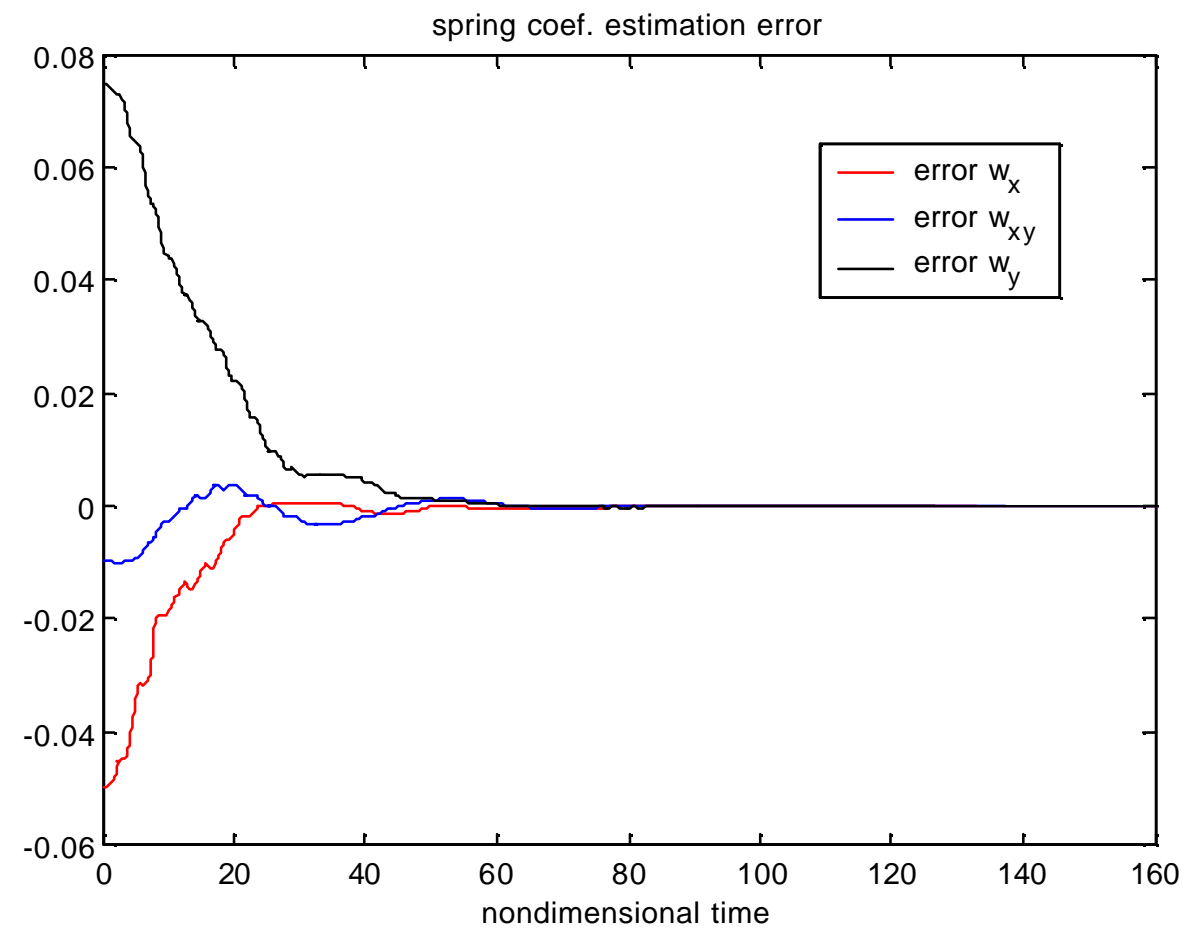

(b)

Figure 8. Parameter estimation errors:

(a) damping coefficients, (b) spring coefficients 


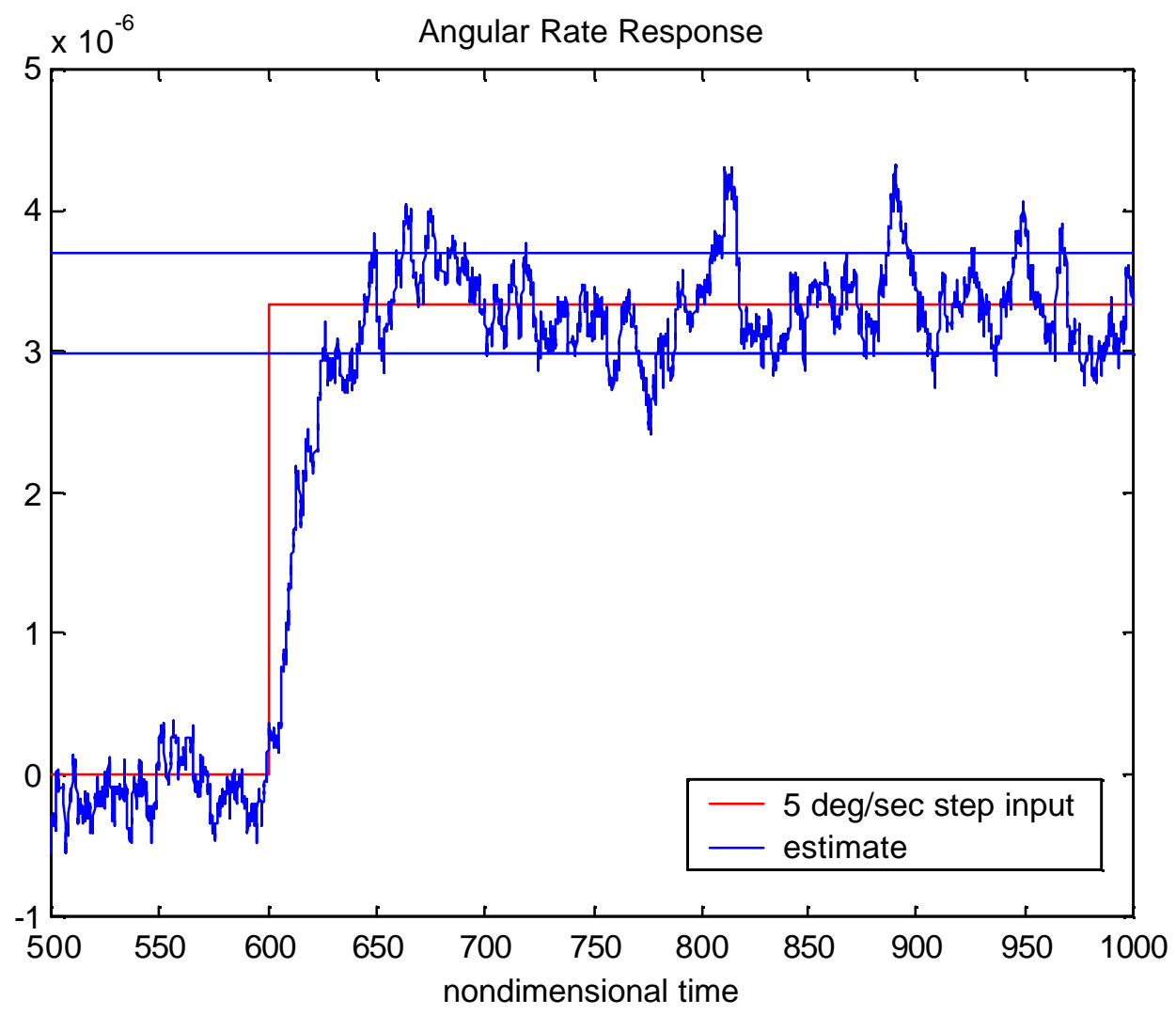

Figure 9. Time responses of angular rate estimate to the $5 \mathrm{deg} / \mathrm{sec}$ step input 


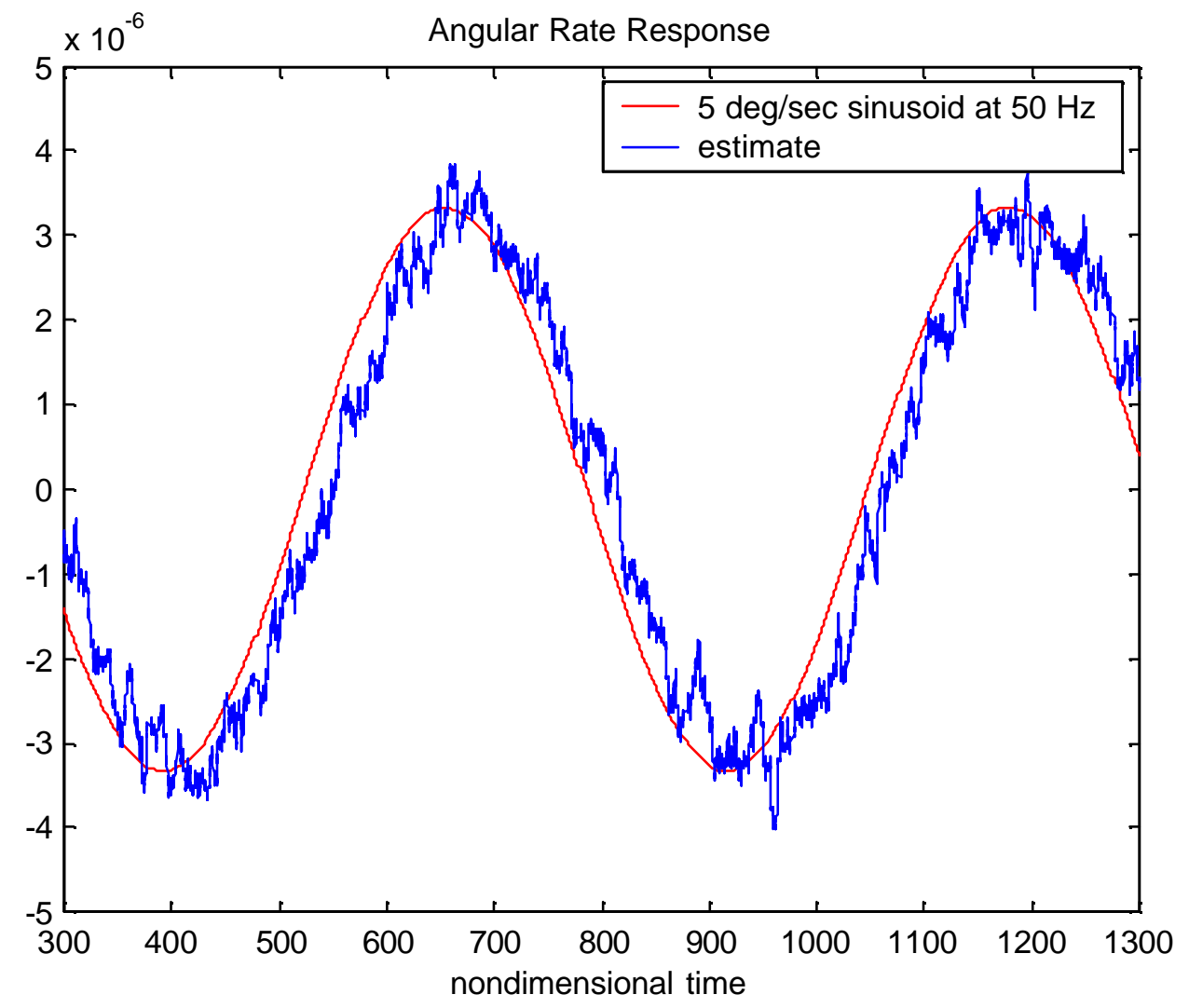

Figure 10. Time responses of angular rate estimate to the $5 \mathrm{deg} / \mathrm{sec}$ sinusoid input at $50 \mathrm{~Hz}$ 